\title{
The Activity of the HF-Modified Ferrospheres in Oxidative Coupling of Methane
}

\author{
Nadezhda P. Kirik*, Natalia N. Anshits, \\ Evgenii V. Rabchevskii and Leonid A. Solovyov \\ Institute of Chemistry and Chemical Technology SB RAS \\ FRC "Krasnoyarsk Science Center SB RAS" \\ 50/24 Akademgorodok, Krasnoyarsk, 660036, Russia
}

Received 11.04.2018, received in revised form 07.07.2018, accepted 22.08.2018

Catalytic properties in the reaction of oxidative coupling of methane (OCM) of HF-modified ferrospheres separated from combustion brown coal fly ash are investigated. It is established that the modification of ferrospheres results in a significant increase in the efficiency of $C_{2}$-hydrocarbons formation at $750{ }^{\circ} \mathrm{C}$, the yield increases by 1.5-2.0 times, the content of ethylene in the $C_{2}$-products up to $30 \%$, and at $850{ }^{\circ} \mathrm{C}-$ up to $65 \%$. It is suggested that the high efficiency of ethylene formation is associated with the formation of oxifluoride centers, which are deactivated at $850{ }^{\circ} \mathrm{C}$ and converted into oxide centers of deep oxidation.

Keywords: fly ash ferrospheres, HF-modification, oxidative coupling of methane.

Citation: Kirik N.P., Anshits N.N., Rabchevskii E.V., Solovyov L.A. The activity of the HF-modified ferrospheres in oxidative coupling of methane, J. Sib. Fed. Univ. Chem., 2018, 11(3), 347-360. DOI: 10.17516/1998-2836-0081.

(c) Siberian Federal University. All rights reserved

* Corresponding author E-mail address: kiriknp@icct. ru 


\title{
Активность НF-модифицированных ферросфер \\ в окислительной конденсации метана
}

\author{
Н.П. Кирик, Н.Н. Аншиц, \\ Е.В. Рабчевский, Л.А. Соловьев \\ Институт химии и химической технологии СО РАН \\ ФИЦ «Красноярский научный иентр СО РАН» \\ Россия, 660036, Красноярск, Академгородок, 50/24
}

Исследованы каталитические свойства в реакиии окислительной конденсаџии метана (ОКМ) HF-модифичированных ферросфер, выделенных из летучей золы от сжигания бурого угля. Установлено, что модифицирование ферросфер приводит к существенному увеличению эффективности образования $C_{2}$-углеводородов: при $750{ }^{\circ} \mathrm{C}$ выход возрастает в 1,5-2,0 раза, доля этилена в $C_{2}$-продуктах - до $30 \%$, а при $850{ }^{\circ} \mathrm{C}-$ до $65 \%$. Высокая эффективность образования этилена связана с формированием оксифторидных иентров, которые при $850{ }^{\circ} \mathrm{C}$ дезактивируются и превращаются в оксидные центры глубокого окисления.

Ключевые слова: ферросферы летучих зол, НF-модифицирование, окислительная конденсация метана.

\section{Введение}

Реакция окислительной конденсации метана (ОКМ) с образованием $\mathrm{C}_{2}$-углеводородов является привлекательным способом превращения наиболее инертного в природном газе углеводорода - метана - в ценные химические продукты, в первую очередь в этилен. К настоящему времени в качестве катализаторов этой реакции исследованы оксиды различных элементов и сложные композиции на их основе. Основная часть работ посвящена индивидуальным и модифицированным оксидам щелочных, щелочно- и редкоземельных металлов и $\mathrm{Mn}$-содержащим контактам [1-3]. Исследовалось влияние модифицирования как катионной, так и анионной подрешетки оксидов на их активность и селективность. Установлено, что использование катализаторов, промотированных галогенидами щелочных и щелочноземельных металлов, приводит к повышению их эффективности в образовании $\mathrm{C}_{2}$-углеводородов за счет роста конверсии метана либо значительного возрастания селективности по $\mathrm{C}_{2}$-продуктам, в которых часто наблюдается увеличение доли этилена [4-11]. Аналогичные эффекты наблюдаются при введении хлорсодержащего компонента в газовую фазу при проведении процесса в присутствии оксидных контактов $[12,13]$.

Гетерогенно-гомогенный механизм процесса ОКМ на оксидных катализаторах включает взаимодействие молекул метана с атомарными формами кислорода, стабилизированными на дефектах структуры, с формированием $\mathrm{CH}_{3}$-радикалов и их последующей рекомбинацией в газовой фазе с образованием этана. Этилен образуется в результате дегидрирования этана с участием тех же активных форм кислорода. Модифицирование оксидов галогенидами изменяет природу активных центров. Так, для Cl-модифицированных оксидов предполагается 
образование дефектов за счет замещения иона кислорода ионом хлора: в $\mathrm{Li} / \mathrm{MgO}$-катализаторе с образованием дефектов $\left[\mathrm{Li}^{+} \mathrm{Cl}-\right]$, в щелочно- и редкоземельных оксидах с образованием оксихлоридных комплексов [4-5, 14-17]. Важно отметить, что монофазные хлориды щелочноземельных элементов $\left(\mathrm{CaCl}_{2}, \mathrm{SrCl}_{2}, \mathrm{BaCl}_{2}\right)$, а также фториды щелочно- и редкоземельных элементов $\left(\mathrm{SrF}_{2}, \mathrm{BaF}_{2}, \mathrm{LaF}_{3}\right)$ проявляют низкую активность в превращении метана и не селективны в образовании этилена $[8,17,18]$. Эффективность в ОКМ галогенидсодержащих двухкомпонентных систем $\mathrm{BaX}_{2} /$ редкоземельный оксид $(\mathrm{X}=\mathrm{F}, \mathrm{Cl}, \mathrm{Br})$ зависит от природы как оксида, так и галогенида. Процессы замещения ионов в катионной и анионной подрешетках приводят к формированию дефектных центров, которые более эффективны в активировании С-Н-связи в метане [9]. Для контактов $\mathrm{BaX}_{2} / \mathrm{La}_{2} \mathrm{O}_{3}(\mathrm{X}=\mathrm{F}, \mathrm{Br})$, из которых более эффективным является $\mathrm{BaBr}_{2} / \mathrm{La}_{2} \mathrm{O}_{3}$, установлено, что ион $\mathrm{Br}$ с большим радиусом по сравнению с $\mathrm{O}^{2-}$ связывается поверхностью $\mathrm{La}_{2} \mathrm{O}_{3}$, а ион $\mathrm{F}^{-}$диффундирует в объем с образованием $\mathrm{LaF}_{3}$ и $\mathrm{LaOF}$. При $750{ }^{\circ} \mathrm{C}$ отношение этилена к этану в продуктах на системах $10 \% \mathrm{BaF}_{2} / \operatorname{Ln}_{2} \mathrm{O}_{3}(\mathrm{Ln}=\mathrm{Y}, \mathrm{La})$ и $10 \% \mathrm{BaBr}_{2} /$ $\mathrm{La}_{2} \mathrm{O}_{3}$ составляет 1,03, 1,25 и 2,11 соответственно [8]. При более высокой эффективности в процессе ОКМ по сравнению с оксидными катализаторами исследованные Сl-модифицированные системы имеют существенный недостаток, связанный со снижением их активности во времени за счет потери хлора $[1,17]$.

Поиск недорогих и термостабильных катализаторов, селективных в образовании этилена, остается актуальной задачей. Фториды и оксифторидные комплексы по сравнению с хлоридными и бромидными более устойчивы в условиях проведения процесса ОКМ. Ранее было показано [19], что после обработки концентрированной HF катализатора на основе ферросфер летучих зол от пылевидного сжигания бурого ирша-бородинского угля с содержанием $\mathrm{Fe}_{2} \mathrm{O}_{3}$ 87,5 мас. \% конверсия метана возрастает с 9,5 до $18 \%$, а селективность по $\mathrm{C}_{2}$-продуктам - с 61 до 67 \%. Обработку ферросфер кислотой проводили с целью увеличения доступной активной поверхности за счет вытравливания алюмосиликатной компоненты. Данные по исследованию катализатора после катализа методами ЯГР и РФЭС показали, что наряду с удалением стеклофазы происходит также модифицирование ферросфер с образованием нового типа центров, содержащих фтор. Целью данной работы является детальное исследование влияния HF-модифицирования катализаторов на основе ферросфер, выделенных из летучих зол от сжигания березовского бурого угля, с содержанием $\mathrm{Fe}_{2} \mathrm{O}_{3}$ 84-98 мас. \% на их фазовый состав и каталитические свойства в реакции окислительной конденсации метана.

\section{Экспериментальная часть}

\section{Получение катализаторов}

Исходные образиы ферросфер получали из двух фракций высококальциевых ферросфер, SMF -0,160+0,100 мм (90,36 мас. \% $\left.\mathrm{Fe}_{2} \mathrm{O}_{3}\right)$ и $\mathrm{SMF}-0,100+0,063$ мм (90,85 мас. \% $\left.\mathrm{Fe}_{2} \mathrm{O}_{3}\right)$, с помощью дополнительной магнитной классификации в градиентном магнитном поле с использованием магнитного сепаратора 138 Т (Россия). Фракции ферросфер были выделены из летучих зол от сжигания угля Березовского месторождения (Красноярский край). Детальное описание способа получения узких фракций ферросфер и определения их физико-химических характеристик приведено в работе [20]. Из каждой фракции ферросфер при магнитной сепарации было получено по четыре образца. В табл. 1 приведены значения удельной поверхности и данные хи-

$$
-349-
$$




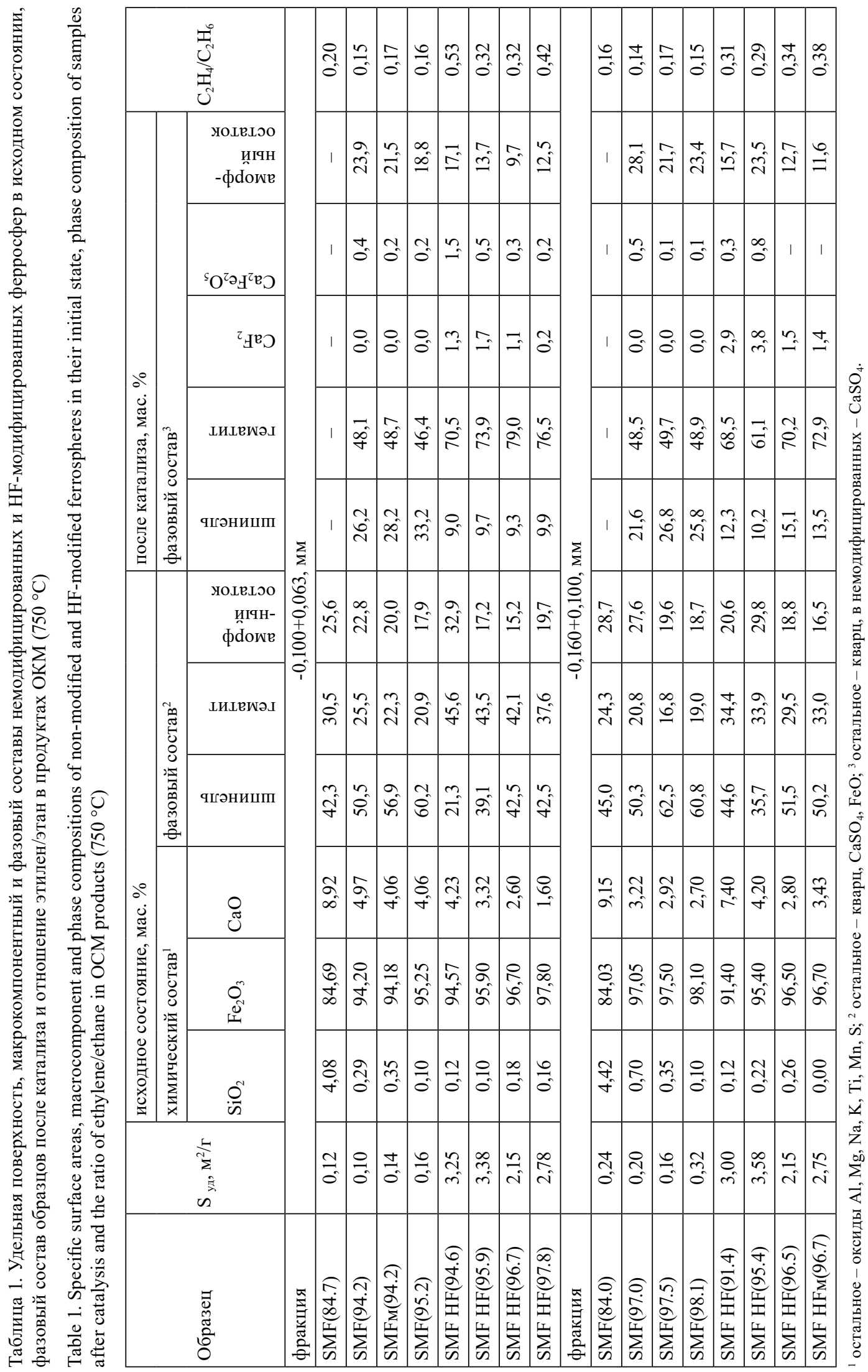


мического анализа (содержание оксидов основных элементов) для полученных образцов, обозначенных как $\mathrm{SMF}(\mathrm{n})$, где в скобках приведено массовое содержание $\mathrm{Fe}_{2} \mathrm{O}_{3}$. Из данных табл. 1 видно, что полученные исходные образцы, за исключением $\operatorname{SMF}(84.0)$ и $\operatorname{SMF}(84.7)$, представляют оксидную железо-кальциевую систему с содержанием суммы оксидов железа и кальция не меньше 98,0 мас. \%.

HF-модифицированные ферросферы получали обработкой исходных образцов ферросфер концентрированной плавиковой кислотой (марка «х. ч.») в полипропиленовой емкости в следующих условиях: соотношение жидкая фаза (мл)/твердая фаза (г) = 5, длительность обработки 10 мин, периодическое перемешивание. С целью удержания магнитной части образца при декантации травильного раствора пропиленовую емкость устанавливали на постоянный магнит. Продукт промывали дистиллированной водой до отрицательной качественной реакции на фтор-ионы с хлоридом бария $\left(0,1\right.$ н) и сушили при температуре $(110 \pm 5){ }^{\circ} \mathrm{C}$ до постоянной массы.

При обработке ферросфер HF происходит разрушение глобул, а удельная поверхность образцов увеличивается больше, чем на порядок (табл. 1). После стадии сушки проводили контрольный рассев образца на ситах с ячейками, соответствующими размеру исходной фракции. Потери после всех стадий составляли 45-74 мас. \% от исходной фракции. По данным химического анализа, содержание Fе и Са в пересчете на оксиды в HF-модифицированных образцах ферросфер составляет не менее 98 мас. \% (табл. 1). Модифицированные образцы обозначены как SMF HF(n), где в скобках отражено массовое содержание $\mathrm{Fe}_{2} \mathrm{O}_{3}$. При монотонном снижении в образцах содержания $\mathrm{Fe}_{2} \mathrm{O}_{3}$ в интервале 97,8-91,4 мас. \% наблюдается рост содержания $\mathrm{CaO}$ в интервале 1,6-7,4 мас. \%. В качестве примера на рис. 1 приведен микроснимок образца SMF HFм (96.7).

\section{Каталитические измерения}

Каталитические свойства полученных образцов в реакции ОКМ изучали в проточной установке со стационарным слоем катализатора в кварцевых трубчатых микрореакторах с внутренним диаметром 4-8 мм при температурах $750-850{ }^{\circ} \mathrm{C}$, в составе реакционной смеси $\mathrm{CH}_{4}: \mathrm{O}_{2}: \mathrm{He}=80: 10: 10$ (об. \%) и атмосферном давлении. Навеска образцов равнялась 0,3-1,3 г, нагрузка $-1,2-1,3$ г-кат.с/мл. Состав исходной смеси и продуктов реакции анализировали на

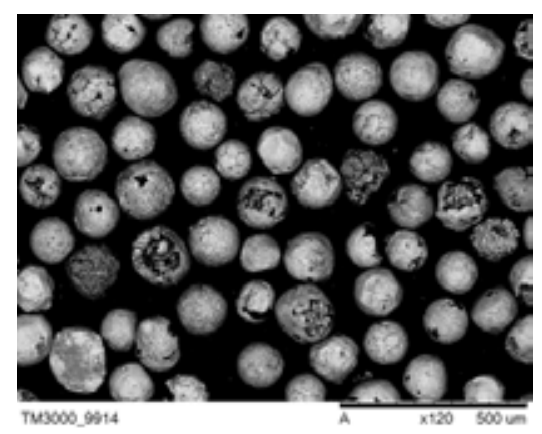

Рис. 1. СЕМ-снимок HF-модифицированного образца SMF HFм(96.7) в исходном состоянии

Fig. 1. SEM image of HF-modified sample SMF $\mathrm{HFM}(96.7)$ in its initial state 
хроматографе Agilent 7890A GC (США), оснащенном набивными колонками HP Plot $\mathrm{Al}_{2} \mathrm{O}_{3}$, Molecular Sieve 5A и капиллярной колонкой DB-1. Относительная ошибка определения концентраций газовых компонентов составляла $\pm 5 \%$. Каталитические свойства образцов определяли через 4 ч от начала проведения эксперимента.

\section{Физико-химические методы исследования катализаторов}

Удельную поверхность образцов определяли методом многоточечной БЭТ по низкотемпературной адсорбции азота на сорбционном анализаторе NOVA 3200e (Quantachrome Instruments, USA).

Химический состав полученных образцов был определен по ГОСТ 5382-91.

Количественный фазовый состав образцов в исходном состоянии и после проведения катализа определен с помощью порошкового рентгенофазового анализа с применением полнопрофильного метода Ритвельда и метода минимизации производной разности на дифрактометре X'Pert Pro MPD (PANalytical, Netherlands) с твердотельным детектором PIXcel и вторичным графитовым монохроматором для СоК $\alpha$-излучения аналогично [20, 21].

Исследование состава, строения поверхности глобул и их полированных срезов проводили с использованием сканирующего электронного микроскопа TM-3000 (Hitachi, Япония) (СЭМ), оборудованного системой микроанализа Quantax70 с энергодисперсионным спектрометром Bruker XFlash 430Н (Германия) (ЭДС). Время накопления определялось по качеству сборки спектра, позволяющему проводить количественную обработку, и было не менее 10 мин.

\section{Результаты и их обсуждение}

\section{Каталитические свойства $H F$-модифицированных ферросфер}

При проведении процесса ОКМ при $750{ }^{\circ} \mathrm{C}$ в присутствии как исходных, так и HFмодифицированных образцов ферросфер основными продуктами являются $\mathrm{C}_{2}$-углеводороды этан и этилен, и оксиды углерода - $\mathrm{CO}$ и $\mathrm{CO}_{2}$. Катализаторы на основе исходных ферросфер, как было показано ранее, проявляют стабильную во времени активность [19, 22]. Изменение селективностей образования основных продуктов - суммы $\mathrm{C}_{2}$-углеводородов и $\mathrm{CO}_{2}$, в присутствии HF-модифицированных ферросфер в течение 4-х ч проведения процесса приведено на рис. 2 .

Видно, что начальная селективность образования $\mathrm{C}_{2}$-продуктов образцов находится на уровне 70-80 \% и со временем несколько снижается. Через 4 ч проведения процесса снижение селективности составляет не более $10 \%$, а в течение последнего часа - не более 1,5 \%. Изменение селективности по $\mathrm{CO}_{2}$ носит противоположный характер: начальные значения составляют 12-24 \%, через 4 ч возрастают до 23-33 \%. Исключением является образец с высоким содержанием железа, SMF HF(97.8), в присутствии которого в начальный период наблюдается небольшой, на 2,5 \%, рост селективности по $\mathrm{C}_{2}$-углеводородам, которая далее не меняется. Для сопоставления каталитических свойств образцов использовали данные после 4 ч проведения эксперимента, приняв, что катализаторы находятся в квазистационарном состоянии. На рис. 3 приведены зависимости селективностей образования суммы $\mathrm{C}_{2}$-углеводородов от конверсии метана в присутствии немодифицированных и HF-модифицированных образцов при $750{ }^{\circ} \mathrm{C}$. 


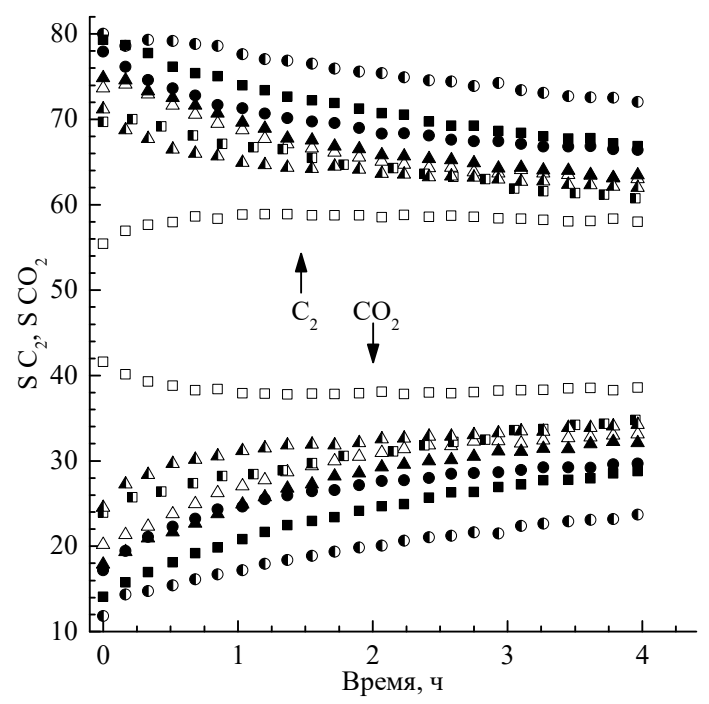

Рис. 2. Изменение во времени селективностей образования суммы $\mathrm{C}_{2}$-углеводородов и $\mathrm{CO}_{2}$ в присутствии HF-модифицированных ферросфер, $750{ }^{\circ} \mathrm{C}: \square-\operatorname{SMF~HF(97.8);~-~} \operatorname{SMF} \operatorname{HF}(94.6) ; \Delta-\operatorname{SMF} \operatorname{HF}(96.7) ; \Delta-$ SMF HF(96.5); $\boldsymbol{\Delta}$ - SMF HFM(96.7); • - SMF HF(95.9); - - SMF HF(91.4); - SMF HF(95.4)

Fig. 2. Selectivity to $\mathrm{C}_{2}$-hydrocarbons and $\mathrm{CO}_{2}$ over HF-modified ferrospheres versus time; $750{ }^{\circ} \mathrm{C}: \square-\mathrm{SMF}$ HF(97.8); $\mathbf{D}$ - SMF HF(94.6); $\Delta$ - SMF HF(96.7); $\Delta$ - SMF HF(96.5); $-\operatorname{SMF~HFM(96.7);~•~-~SMF~HF(95.9);~}$ - $-\mathrm{SMF} \mathrm{HF}(91.4) ; 0-\mathrm{SMF} \mathrm{HF}(95.4)$

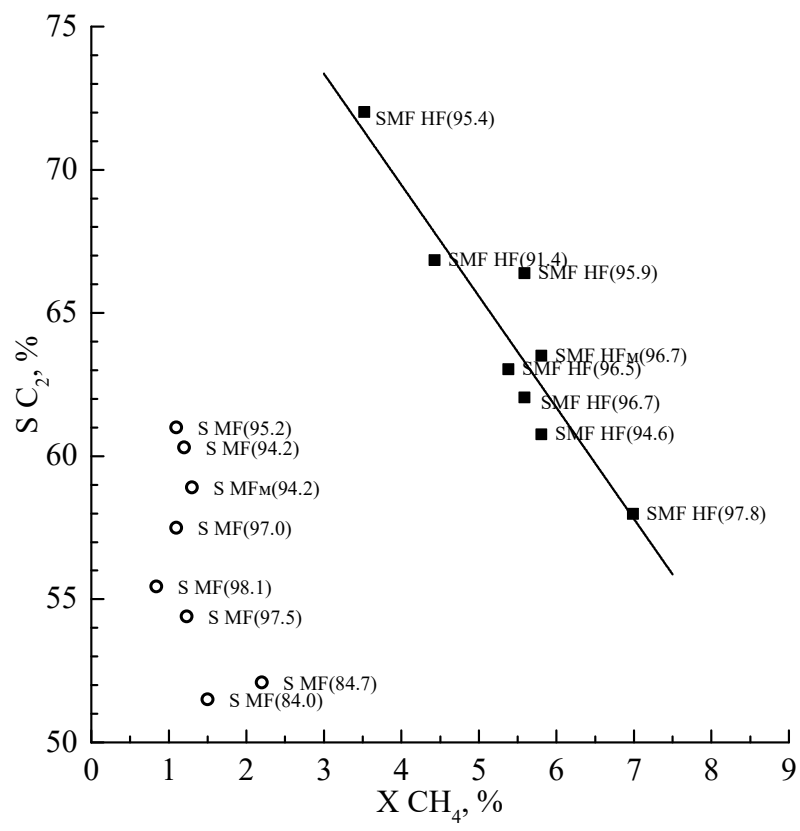

Рис. 3. Зависимость селективностей образования суммы $\mathrm{C}_{2}$-углеводородов от конверсии метана, $750{ }^{\circ} \mathrm{C}$ : о - немодифицированные ферросферы; - - НF-модифицированные ферросферы

Fig. 3. The dependence of selectivity to $\mathrm{C}_{2}$-hydrocarbons on $\mathrm{CH}_{4}$ conversion, $750{ }^{\circ} \mathrm{C}$ : $\circ-$ non-modified ferrospheres; - - HF-modified ferrospheres 
С увеличением конверсии метана наблюдается снижение $\mathrm{C}_{2}$-селективности, что согласуется с общей закономерностью изменения селективности по $\mathrm{C}_{2}$-продуктам от конверсии метана, установленной для катализаторов ОКМ [23]. В исследованных условиях исходные ферросферы с содержанием $\mathrm{Fe}_{2} \mathrm{O}_{3}$ 94-98 мас. \% характеризуются низкой конверсией метана, 0,7-1,2 \%, и $\mathrm{C}_{2}$ селективностью в интервале 54-61 \%. Образцы с меньшим содержанием $\mathrm{Fe}_{2} \mathrm{O}_{3}$ (84-85 мас. \%) проявляют более высокую конверсию метана, 1,5-2,2 \%, при меньшей селективности 51-52 \%. Селективность образования СО на всех образцах варьируется в интервале 9-14 \%.

Из приведенных на рис. 3 данных видно, что для HF-модифицированных ферросфер наблюдается резкое, до $3,5-7,0 \%$, увеличение конверсии метана и рост $\mathrm{C}_{2}$-селективности до 58 $72 \%$. Селективность образования СО на всех модифицированных образцах лежит в интервале 3-4 \%, который соответствует литературным данным для фторсодержащих катализаторов $\left(\mathrm{SrF}_{2}\right.$ и $\mathrm{BaF}_{2}$, нанесенные на редкоземельные оксиды) $[8,18]$. Модифицированные ферросферы, кроме более высокой $\mathrm{C}_{2}$-селективности, существенно отличаются от исходных образцов большей долей этилена в $\mathrm{C}_{2}$-продуктах. Соотношение $\mathrm{C}_{2} \mathrm{H}_{4} / \mathrm{C}_{2} \mathrm{H}_{6}$ для $\mathrm{HF}$-модифицированных систем составляет 0,29-0,53, тогда как для исходных лежит в интервале 0,14-0,20 (табл. 1). Наблюдаемые различия в каталитических свойствах исходных и HF-модифицированных ферросфер (рис. 3, табл. 1) трудно объяснить только бо́льшим значением удельной поверхности модифицированных образцов. При проведении ОКМ в присутствии НF-модифицированных ферросфер наряду с увеличением конверсии метана наблюдается также возрастание и селективности образования $\mathrm{C}_{2}$-углеводородов, и доли этилена в $\mathrm{C}_{2}$-продуктах, что указывает на различие в природе активных центров.

Увеличение активности оксидов и их селективности в образовании этилена при модифицировании галогенами связывают с двумя факторами: формированием нового типа активных оксигалогенидных центров и/или фаз и с подавлением центров глубокого окисления. В присутствии хлоридсодержащих катализаторов в условиях процесса происходит генерация хлоррадикалов, участвующих в активации метана. Предполагается также возможность протекания стадии дегидрирования этана с участием активных оксихлоридных центров [4-5, 14-16]. При использовании фтормодифицированных оксидов образование фторрадикалов исключается и изменение каталитических свойств в ОКМ связывают с образованием более эффективных в активировании кислорода оксифторидных структур [9, 24, 25]. Так, в работе [25] показано, что в нанесенных $\mathrm{BaF}_{2}, \mathrm{SrF}_{2}$ на редкоземельные оксиды катализаторах наблюдается частичный ионный обмен $\mathrm{O}^{2-} / \mathrm{F}^{-}$, что приводит к образованию анионных вакансий и новой оксифторидной фазы с дефектной структурой. С целью определения влияния НF-модифицирования на структурные характеристики ферросфер был исследован фазовый состав образцов в исходном состоянии и после проведения катализа.

\section{Фазовый состав HF-модифицированных ферросфер}

Данные по содержанию основных кристаллических фаз в исходных и HF-модифицированных ферросферах до и после катализа приведены в табл. 1. Основными железосодержащими фазами в исходных ферросферах являются фазы феррошпинели и гематита. С увеличением содержания $\mathrm{Fe}_{2} \mathrm{O}_{3}$ в образце количество фазы шпинели возрастает с 42,3 до 62,5 мас. \%, а фазы гематита снижается с 30,5 до 16,8 мас. \%. Параметр решетки шпинели во всех исследованных 
в работе исходных образцах, как и в исследованных ранее ферросферах более мелких фракций [21], лежит в интервале 8.398-8.399 А̊ и превышает значение для стехиометрического магнетита $(8,396 \AA$ А̊). Более высокое по сравнению со стехиометрическим магнетитом значение параметра феррошпинельной фазы в образцах ферросфер свидетельствует о формировании решетки с изоморфным замещением железа на кальций $[20,26]$. Содержание аморфного остатка в исходных ферросферах до и после катализа практически не меняется и варьируется от 18 до 29 мас. \%. В образцах после катализа наблюдается увеличение содержания гематита до 46,449,7 мас. \% и снижение феррошпинели, до 33,2-21,6 мас. \%, что хорошо согласуется с ранее полученными данными [21].

Фазовый состав HF-модифицированных ферросфер показывает, что в исходном состоянии (до катализа) качественных изменений состава по сравнению с немодифицированными образцами не происходит, но образцы содержат больше фазы гематита, 29,5-45,6 мас. \%, и меньше фазы шпинели, 51,5-21,3 мас. \% (табл. 1). Значение параметра решетки шпинельной фазы в модифицированных ферросферах варьируется в интервале 8.397-8.401 А̊. После катализа в модифицированных образцах, как и в исходных, содержание гематита возрастает до 61,1-79,0 мас. \%, а содержание шпинели снижается до 15,1-9,0 мас. \%. В отличие от исходных ферросфер наблюдается новая фаза $\mathrm{CaF}_{2}$, содержание которой варьируется от 0,2 до 3,8 мас. \%. Содержание аморфного остатка в модифицированных ферросферах после катализа снижено, по-видимому, вследствие процесса раскристаллизации, и варьируется от 10 до 23 мас. \% по сравнению с образцами в исходном состоянии, в которых он равен 15-33 мас. \%.

В результате СЭМ-ЭДС-исследования исходных НF-модифицированных ферросфер, SMF $\mathrm{HF}(97.8)$ и SMF HF(95.4), установлено, что на поверхности большинства глобул ферросфер и их полированных срезов концентрации $\mathrm{Fe}_{2} \mathrm{O}_{3}, \mathrm{CaO}$ и $\mathrm{F}$ варьируются в пределах 63-98, 0,3-19 и 0,1-6 мас. \% соответственно, что свидетельствует об обогащении поверхности фтором. Отсутствие в этих ферросферах контролируемого количества фторсодержащих фаз объясняется формированием рентгеноаморфных соединений или поверхностных фторжелезооксидных и/ или кальцийфторжелезооксидных комплексов. Появление после катализа фазы $\mathrm{CaF}_{2}$ связано, по-видимому, с протеканием процесса кристаллизации в реакционных условиях и практическим отсутствием при этой температуре процесса пирогидролиза флюорита [27]. Повышенное содержание фазы $\mathrm{Ca}_{2} \mathrm{Fe}_{2} \mathrm{O}_{5}$ в некоторых образцах по сравнению с исходными может быть обусловлено участием гематита в процессе частичного пирогидролиза $\mathrm{CaF}_{2}$. Известно, что пирогидролиз флюорита в присутствии $\mathrm{SiO}_{2}, \mathrm{U}_{3} \mathrm{O}_{8}$ протекает при более низких температурах с образованием силикатов кальция и $\mathrm{CaU}_{2} \mathrm{O}_{7}$. В качестве добавок рекомендуются также другие оксиды - $\mathrm{PbO}, \mathrm{Cr}_{2} \mathrm{O}_{3}, \mathrm{ZrO}_{2}$, включая $\mathrm{Fe}_{2} \mathrm{O}_{3}$ [27]. Как будет показано ниже, между содержанием $\mathrm{CaF}_{2}$ в модифицированных образцах после катализа и выходом продуктов ОКМ наблюдается отрицательная корреляция. Поэтому высокую эффективность в процессе ОКМ HF-модифицированных ферросфер можно объяснить формированием в них нового типа активных центров, включающих ферритные или Са-ферритные оксифторидные комплексы.

\section{Устойчивость активных комплексов}

После проведения каталитических экспериментов с модифицированными образцами наблюдается помутнение стенки кварцевого реактора, которое свидетельствует о присутствии 


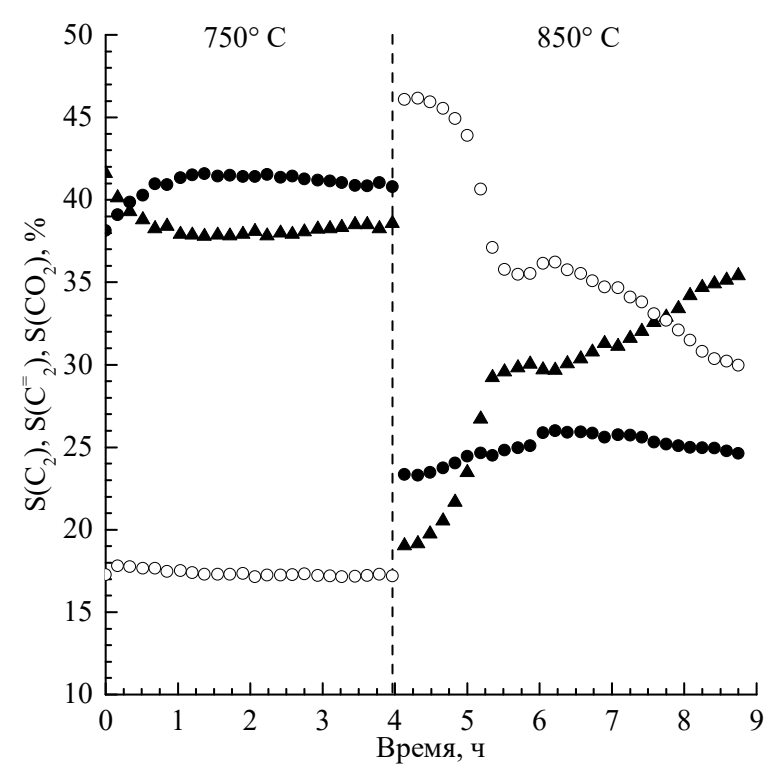

Рис. 4. Изменение селективностей образования продуктов в присутствии $\operatorname{SMF} \mathrm{HF}(97.8)$ при температурах 750 и $850{ }^{\circ} \mathrm{C}$ во времени: • $-\mathrm{C}_{2} \mathrm{H}_{6} ;$ ○ $-\mathrm{C}_{2} \mathrm{H}_{4} ; \boldsymbol{\Delta}-\mathrm{CO}_{2}$

Fig. 4. Selectivity to $\mathrm{C}_{2}$-hydrocarbons over $\mathrm{SMF} \mathrm{HF}(97.8)$ versus time at 750 and $850{ }^{\circ} \mathrm{C}: \bullet-\mathrm{C}_{2} \mathrm{H}_{6} ; \circ-\mathrm{C}_{2} \mathrm{H}_{4}$; $\boldsymbol{\Delta}-\mathrm{CO}_{2}$

фторида водорода в газовой фазе. HF образуется в реакционных условиях за счет разрушения фторсодержащих комплексов в результате их пирогидролиза парами воды. При длительном проведении процесса разрушение активных комплексов должно проявиться в изменении каталитических характеристик модифицированных образцов. Для проверки этого предположения был исследован наиболее активный образец, $\operatorname{SMF~HF(97.8),~при~более~высокой~температуре~-~}$ $850{ }^{\circ} \mathrm{C}$. На рис. 4 приведено изменение селективностей образования этана, этилена и $\mathrm{CO}_{2}$ при температурах 750 и $850{ }^{\circ} \mathrm{C}$. При $750{ }^{\circ} \mathrm{C}$ значения селективностей $\mathrm{SC}_{2}, \mathrm{SC}_{2}=\mathrm{SCO}_{2}$ со временем мало меняются и через 4 ч составляют $41,17,38 \%$ соответственно, соотношение $\mathrm{C}_{2} \mathrm{H}_{4} / \mathrm{C}_{2} \mathrm{H}_{6}$ в продуктах равно 0,41 . Повышение температуры до $850{ }^{\circ} \mathrm{C}$ приводит к изменению селективностей по всем продуктам, в начальный момент селективности имеют значения: $\mathrm{SC}_{2} 23 \%, \mathrm{SC}_{2}=$ $46 \%, \mathrm{SCO}_{2} 19 \%$, соотношение $\mathrm{C}_{2} \mathrm{H}_{4} / \mathrm{C}_{2} \mathrm{H}_{6}$ возрастает до 2,0 . Кроме того, в продуктах появляются также $\mathrm{C}_{3}$-углеводороды, пропан и пропилен, с селективностями 0,5 и $6 \%$. Через 40 мин протекания процесса селективность по этилену резко снижается с 46 до 36 \%, а селективность по $\mathrm{CO}_{2}$ возрастает с 19 до 30 \%. В течение последующих четырех часов изменение селективностей носит монотонный характер. Селективность образования $\mathrm{C}_{2} \mathrm{H}_{6}$ во время всего эксперимента составляет 23-24 \%. Исследование фазового состава образца после катализа при температуре $850{ }^{\circ} \mathrm{C}$ показало, что содержание $\mathrm{CaF}_{2}$ становится меньше, $<0,1$ мас. \%, а содержание фазы $\mathrm{Ca}_{2} \mathrm{Fe}_{2} \mathrm{O}_{5}$ возрастает до 0,9 мас. $\%$ (при $750{ }^{\circ} \mathrm{C}$ содержание фаз составляло 0,2 и 0,2 мас. \%, табл. 1).

$\mathrm{C}$ ростом содержания $\mathrm{CaF}_{2}$ в образцах после катализа при $750{ }^{\circ} \mathrm{C}$, как видим из данных, приведенных на рис. 5 , наблюдается снижение выходов $\mathrm{C}_{2}$-продуктов и $\mathrm{CO}_{2}$, при этом селективность по $\mathrm{C}_{2}$-продуктам, за счет более резкого снижения выхода $\mathrm{CO}_{2}$ возрастает, а доля этилена

$$
-356-
$$




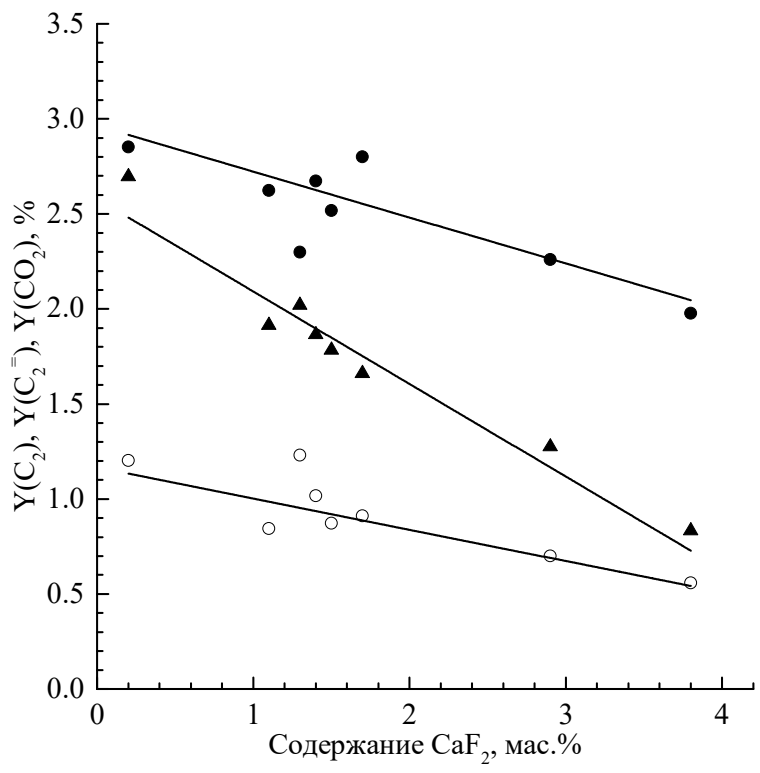

Рис. 5. Изменение выходов образования этана, этилена и диоксида углерода от содержания $\mathrm{CaF}_{2}$ в $\mathrm{HF}-$ модифицированных образцах после катализа, $750{ }^{\circ} \mathrm{C}: \bullet-\mathrm{C}_{2} \mathrm{H}_{6} ; \circ-\mathrm{C}_{2} \mathrm{H}_{4} ; \boldsymbol{\Delta}-\mathrm{CO}_{2}$

Fig. 5. The yield of ethane, ethylene and carbon dioxide versus $\mathrm{CaF}_{2}$ content in $\mathrm{HF}$-modified ferrospheres after catalysis, $750{ }^{\circ} \mathrm{C}: \bullet-\mathrm{C}_{2} \mathrm{H}_{6} ; \circ-\mathrm{C}_{2} \mathrm{H}_{4} ; \boldsymbol{\Delta}-\mathrm{CO}_{2}$

в $\mathrm{C}_{2}$-углеводородах несколько снижается. Результаты согласуются с литературными данными для фторсодержащих контактов ОКМ, в работах $[8,18]$ показано, что при $750{ }^{\circ} \mathrm{C}$ монофазные фториды $\mathrm{SrF}_{2}, \mathrm{BaF}_{2}, \mathrm{LaF}_{3}$ проявляют низкую активность в ОКМ (конверсия метана 0,7-2,2 \%, селективность по этану 54 \%) и нулевую селективность в образовании этилена. По данным рис. 3 и 5 можно заключить, что фторид кальция как неактивный в ОКМ компонент блокирует частично поверхность катализатора, что проявляется в снижении конверсии метана и выхода $\mathrm{C}_{2}$-продуктов при повышении селективности их образования при $750{ }^{\circ} \mathrm{C}$. При высокой температуре, $850{ }^{\circ} \mathrm{C}$, фторид кальция выделяя HF поддерживает концентрацию активных в образовании $\mathrm{C}_{2}$-продуктов оксифторидных комплексов и обеспечивает стабильность работы катализатора. В исследованном катализаторе с низким содержанием $\mathrm{CaF}_{2}(0,2$ вес. \%) активные центры начинают разрушаться уже после 40 мин реакции, что приводит к резкому изменению селективностей по $\mathrm{C}_{2} \mathrm{H}_{4}$ и $\mathrm{CO}_{2}$ (рис. 4). Противоположный характер изменения селективностей по $\mathrm{C}_{2} \mathrm{H}_{4}$ и $\mathrm{CO}_{2}$ свидетельствует о протекании стадии дегидрирования этана с участием активных поверхностных центров, которые при $850{ }^{\circ} \mathrm{C}$ дезактивируются с формированием на поверхности катализатора центров глубокого окисления. Разрушение активных оксифторидных комплексов может сопровождаться образованием поверхностных соединений $-\mathrm{Ca}_{2} \mathrm{Fe}_{2} \mathrm{O}_{5}, \mathrm{Fe}_{2} \mathrm{O}_{3}$ и/или $\mathrm{CaO}$. Известно, что контакты на основе феррита кальция и гематита активны в окислении метана, $\mathrm{CO}$, пропилена [28-30], и их формированием можно объяснить возрастающую селективность в образовании $\mathrm{CO}_{2}$ при дезактивации образца.

Таким образом, проведенное исследование показало, что высокая эффективность HFмодифицированных ферросфер обусловлена протеканием процесса ОКМ на оксифторидных активных центрах, образованных с участием ферритных и/или Са-ферритных комплексов.

$$
-357-
$$


Системы на основе HF-модифицированных ферросфер проявляют достаточно стабильную активность и селективность при температуре $750{ }^{\circ} \mathrm{C}$, обычной для процесса ОКМ. Повышение температуры процесса до $850{ }^{\circ} \mathrm{C}$ приводит к дезактивации активных центров образования $\mathrm{C}_{2}$ углеводородов в результате их пирогидролиза парами образующейся воды и к одновременному формированию центров глубокого окисления.

\section{Заключение}

Исследование показало, что модифицирование ферросфер летучих зол от сжигания бурого угля с содержанием $\mathrm{Fe}_{2} \mathrm{O}_{3}$ 84-98 мас. \% концентрированной плавиковой кислотой приводит к получению более эффективных контактов в процессе окислительной конденсации метана. При температуре процесса $750{ }^{\circ} \mathrm{C}$ конверсия метана возрастает в 3,5-5 раз, селективность по $\mathrm{C}_{2}$-продуктам - на 7-11 \% по сравнению с немодифицированными ферросферами. Для всех модифицированных образцов наблюдается увеличение доли этилена в $\mathrm{C}_{2}$-продуктах, начальное отношение $\mathrm{C}_{2} \mathrm{H}_{4} / \mathrm{C}_{2} \mathrm{H}_{6}$ составляет $0,3-0,4$ при $750{ }^{\circ} \mathrm{C}$ (0,1-0,2 - для немодифицированных образцов) и 2,0-2,3 - при $850{ }^{\circ} \mathrm{C}$. Модифицирование приводит к изменению фазового состава образцов, в которых по сравнению с исходными ферросферами содержание фазы гематита возрастает, а феррошпинельной фазы снижается. Изменение каталитических свойств HFмодифицированных ферросфер обусловлено формированием активных центров нового типа, связанных с оксифторидными комплексами, ферритного и/или Са-ферритного состава, которые эффективны в образовании этана и дегидрировании его в этилен. Дезактивация активных комплексов при $850{ }^{\circ} \mathrm{C}$ за счет взаимодействия с парами воды приводит к формированию оксидных центров глубокого окисления, что проявляется в резком снижении селективности по $\mathrm{C}_{2} \mathrm{H}_{4}$ и увеличении селективности по $\mathrm{CO}_{2}$.

\section{Список литературы}

1. Арутюнов В.С., Крылов О.В. Окислительные превращения метана. М.: Наука, 1998. 361 c. [Arutyunov V.S., Krylov O.V. Oxidative Conversion of Methane. Moscow: Nauka, 1998. 361 p. (in Russ.)].

2. Langfeld K., Frank B., Strempel V.E., Berger-Karin C., Weinberg G., Kondratenko E.V., Schomacker R. Comparison of oxidizing agents for the oxidative coupling of methane over state-ofthe-art catalysts. Applied Catalysis A-General 2012. Vol. 417, P. 145-152.

3. Voskresenskaya E.V., Roguleva V.G., Anshits A.G. Oxidant Activation over Structural Defects of Oxide Catalysts in Oxidative Methane Coupling. Catalysis Reviews: Science and Engineering 1995. Vol. 37, P. 101-143.

4. Shigapov A.N., Novozhilova M.A., Vereshchagin S.N., Anshits A.G., Sokolovskii V.D. Peculiarities in Oxidative Conversion of Methane to $\mathrm{C}_{-2}$ Hydrocarbons over $\mathrm{CaO}-\mathrm{CaCl}_{2} \mathrm{Catalysts}$. Reaction Kinetics and Catalysis Letters 1988. Vol. 37(2), P. 397-402.

5. Anshits A.G., Kirik N.P., Roguleva V.G., Shigapov A.N., Selyutin G.E. Oxidative dimerization of methane over $\mathrm{CaO}$ doped with chlorides or alkaline metals. Catalysis Today 1989. Vol. 4(3-4), P. 399-407.

6. Otsuka K., Liu Q., Hatano M., Morikawa A. Synthesis of Ethylene by Partial Oxidation of Methane over the Oxides of Transition-Elements with LiCl. Chemistry Letters 1986. No. 6, P. 903-906. 
7. Minachev Kh.M, Usachev N.Ya., Udut V.N., Khodakov Yu.S. Oxidative Condensation of Methane - a New Pathway to the Synthesis of Ethane, Ethylene, and Other Hydrocarbons. Russian Chemical Reviews 1988. Vol. 57(3), P. 221-233.

8. Au C.T., He H., Lai S.Y., Ng C.F. A comparison of $\mathrm{BaF}_{2} / \mathrm{La}_{2} \mathrm{O}_{3}$ and $\mathrm{BaBr}_{2} / \mathrm{La}_{2} \mathrm{O}_{3}$ catalysts for the oxidative coupling of methane. Journal of Catalysis 1996. Vol. 159(2), P. 280-287.

9. Au C.T., Zhou X.P., Liu Y.W., Ji W.J., Ng C.F. The characterization of $\mathrm{BaF}_{2} / \mathrm{Y}_{2} \mathrm{O}_{3}$ catalysts for the OCM reaction. Journal of Catalysis 1998. Vol. 174(2), P. 153-163.

10. Dai H.X., Liu Y.W., Ng C.F., Au C.T. The performances and characterization of $\mathrm{BaO}-$ and $\mathrm{BaX}_{2}\left(\mathrm{X}=\mathrm{F}, \mathrm{Cl}\right.$, and $\mathrm{Br}$ )-promoted $\mathrm{Y}_{2} \mathrm{O}_{3}$ catalysts for the selective oxidation of ethane to ethene. Journal of Catalysis 1999. Vol. 187(1), P. 59-76.

11. Hiyoshi N., Ikeda T. Oxidative coupling of methane over alkali chloride-Mn- $\mathrm{Na}_{2} \mathrm{WO}_{4} / \mathrm{SiO}_{2}$ catalysts: Promoting effect of molten alkali chloride. Fuel Processing Technology 2015. Vol. 133, P. 29-34.

12. Burch R., Squire G.D., Tsang S.C. Role of Chlorine in Improving Selectivity in the Oxidative Coupling of Methane to Ethylene. Applied Catalysis 1989. Vol. 46(1), P. 69-87.

13. Hiyoshi N., Sato K. Oxidative coupling of methane over $\mathrm{Mn}-\mathrm{Na}_{2} \mathrm{WO}_{4} / \mathrm{SiO}_{2}$ catalyst with continuous supply of alkali chloride vapor. Fuel Processing Technology 2016. Vol. 151, P. 148-154.

14. Arndt S., Laugel G., Levchenko S., Horn R., Baerns M., Scheffler M., Schlogl R., Schomacker R. $A$ Critical Assessment of $\mathrm{Li} / \mathrm{MgO}$-Based Catalysts for the Oxidative Coupling of Methane. Catalysis Reviews-Science and Engineering 2011. V. 53(4), P. 424-514.

15. Burch R., Chalker S., Loader P., Rice D.A., Webb G. Influence of the Presence of Chlorine on the Selectivity to Ethane and Ethene during Methane Coupling over Samarium-Based Catalysts. Applied Catalysis 1991. Vol. 79(2), P. 265-279.

16. Mamedov A.K., Guliev I.A., Shiryaev P.A., Shashkin D.P. Study of the phase composition and structure of sodium-manganese chloride catalysts in oxidative dehydrogenation of ethane. Kinetics and Catalysis 1991. Vol. 31(5 pt2), P. 1064-1068.

17. Baldwin T.R., Burch R., Crabb E.M., Squire G.D., Tsang S.C. Oxidative Coupling of Methane over Chloride Catalysts. Applied Catalysis. 1989. Vol. 56, P. 219-229.

18. Long R., Zhou S., Huang Y., Weng W., Wan H., Tsai K. Promoting effect of F- on Sr/La oxide catalysts for the oxidative coupling of methane. Applied Catalysis A: General 1995. Vol. 133, P. 269280.

19. Fomenko E.V., Kondratenko E.V., Sharonova O.M., Plekhanov V.P., Koshcheev S.V., Boronin A.I., Salanov A.N., Bajukov O.A., Anshits A.G. Novel microdesign of oxidation catalysts. Part 2. The influence of fluorination on the catalytic properties of glass crystal microspheres. Catalysis Today 1998. Vol. 42(3), P. 273-277.

20. Sharonova O.M., Anshits N.N., Solovyov L.A., Salanov A.N., Anshits A.G. Relationship between composition and structure of globules in narrow fractions of ferrospheres. Fuel 2013. Vol. 111, P. 332-343.

21. Anshits A.G., Bayukov O.A., Kondratenko E.V., Anshits N.N., Pletnev O.N., Rabchevskii E.V., Solovyov L.A. Catalytic properties and nature of active centers of ferrospheres in oxidative coupling of methane. Applied Catalysis A-General 2016. Vol. 524, P. 192-199. 
22. Vereshchagin S.N., Rabchevskii E.V., Anshits N.N., Solov'ev L.A., Anshits A.G., Kondratenko E.V. New approach to the preparation of catalysts for the oxidative coupling of methane. Kinetics and Catalysis 2012. Vol. 53(4), P. 449-455.

23. Zavyalova U1., Holena M., Schlögl R., Baerns M. Statistical Analysis of Past Catalytic Data on Oxidative Methane Coupling for New Insights into the Composition of High-Performance Catalysts. ChemCatChem. 2011. Vol. 3, P. 1935-1947.

24. Long R., Luo J., Chen M., Wan H. Oxidative coupling of methane over BaF2-promoted rare earth oxides with variable valence. Applied Catalysis A: General 1997. Vol. 159, P. 171-185.

25. Wan H., Zhou X., Weng W., Long R., Chao Z., Zhang W., Chen M., Luo J., Zhou S. Catalytic performance, structure, surface properties and active oxygen species of the fluoride-containing rare earth (alkaline earth)-based catalysts for the oxidative coupling of methane and oxidative dehydrogenation of light alkanes. Catalysis Today 1999. Vol. 51, P. 161-175.

26. Orewczyk J. Model studies of doped iron oxides. Reduction process of magnetite doubly doped with calcium and magnesium. Journal of Thermal Analysis and Calorimetry 2000. Vol. 60, P. 265-269.

27. Раков Э.Г., Тесленко В.В. Пирогидролиз неорганических фторидов. М.: Энергоатомиздат, 1987. 152 с. [Rakov E.G., Teslenko V.V. Pyrohydrolysis of inorganic fluorides. Moscow: Energoatomizdat, 1987. 152 p. (In Russ.)].

28. Боресков Г.К., Поповский В.В., Лебедева Н.Е., Сазонов В.А., Андрушкевич Т.В. Каталитические свойства ферритов. ІІ. Окисление метана. Кинетика и катализ 1970. Т. 11(5), C. 1253-1261. [Boreskov G.K., Popovskii V.V., Lebedeva N.E., Sazonov V.A., Andrushkevich T.V. On the catalytic activity of ferrites. II. Methane oxidation. Kinetics and Catalysis 1970. Vol. 11(5), P. 12531261. (in Russ.)].

29. Isupova L.A., Tsybulay S.V., Kryukova G.N., Budneva A.A., Paukshtis E.A., Litvak G.S., Ivanov V.P., Kolomiichuk V.N., Pavlyukhin Yu.T., Sadykov V.A. Mechanochemical Synthesis and Catalytic Properties of Calcium Ferrite $\mathrm{C}_{2} \mathrm{Fe}_{2} \mathrm{O}_{5}$. Kinetics and Catalysis 2002. Vol. 43(1), P. 122-128.

30. Daisuke Hirabayashi, Takeshi Yoshikawa, Kazuhiro Mochizuki, Kenzi Suzuki, Yoichi Sakai. Formation of brownmillerite type calcium ferrite $\left(\mathrm{Ca}_{2} \mathrm{Fe}_{2} \mathrm{O}_{5}\right)$ and catalytic properties in propylene combustion. Catalysis Letters 2006. Vol. 110(1-2), P. 155-160. 